\title{
Construcción de la competencia investigativa en ingeniería
}

\author{
José Eucario Parra-Castrillón \\ Facultad de Ingeniería, UNISABANETA, Medellín, Colombia. Eucarioparra5@gmail.com
}

\begin{abstract}
Resumen - Un reto para las universidades es el relacionado con la construcción de la competencia investigativa en los estudiantes. Esto es, como llevar a que ellos participen en los proyectos y procesos de investigación, con compromiso y apasionamiento. Además, como lograr que la competencia investigativa haga parte del perfil profesional de los egresados. En el caso de la ingeniería, dos aspectos pueden considerarse como oportunidades: el primero, desde los primeros niveles los estudiantes están interactuando con la experimentación, las matemáticas y la modelación de fenómenos naturales. El segundo, por su naturaleza el perfil de los ingenieros está relacionado con la innovación y el desarrollo tecnológico, lo cual demanda actitudes, actuaciones y conocimientos en la investigación formativa.

Pensando en lo anterior se proponen en este trabajo un modelo para la formación de la competencia investigativa, que incluye seis elementos de competencias, desglosados cada uno comportamientos esenciales y unos lineamientos pedagógicos y curriculares.
\end{abstract}

Palabras Clave - competencia; competencia investigativa; currículo; elementos de competencia; investigación en ingeniería; investigación formativa; formación investigativa, pedagogía.

Recibido para revisar Mayo 25 de 2017, aceptado Agosto 9 de 2017, versión final Agosto 30 de 2017

\section{Construction of the research skill in engineering}

Abstract - A challenge for universities is related to the construction of investigative skill in students. This is how to get them involved in the research projects and processes, with commitment and passion. In addition, how to make the investigative skill part of the professional profile of graduates. In the case of engineering, two aspects can be considered as opportunities: first, from the first levels students are interacting with experimentation, mathematics and the modeling of natural phenomena. The second, by its nature the profile of the engineers is related to innovation and technological development, which demands attitudes, actions and knowledge in the formative research.

Thinking about the above, we propose in this work a model for the formation of investigative competence, which includes six elements of competences, each broken down into essential behaviors and pedagogical and curricular guidelines.

Keywords - curriculum; elements of competence; engineering research; formative research; research skill; research training; pedagogy;skill.

\section{Introducción}

El tema de las competencias investigativas es de marcado interés en la formación universitaria, ya que cada vez con mayor insistencia los sistemas de calidad exigen evidencias sobre proyectos, investigadores, divulgación e impacto en el medio, al considerarse que junto con la docencia y proyección social, la investigación es una función básica de las instituciones de educación superior. Siendo así, las estrategias para la investigación formativa deben considerarse ampliamente, pues desde ahí se va perfilando el ambiente que potencie a la institución en el desarrollo de la investigación.

Según Restrepo [1] "Hablar de investigación formativa es hablar de formación investigativa o del uso de la investigación para formar en la investigación, para aprender a investigar investigando, aunque esta actividad no conduzca necesariamente a descubrimiento de conocimiento nuevo y universal”. Es decir, el objetivo de la formación investigativa es la formación de la competencia investigativa. Para cumplirlo en los ambientes formativos se interrelacionan el método científico y el método activo de aprendizaje, lo que determina la formación de actitudes y habilidades para la indagación continua y sistemática de información [2].

La construcción de la competencia investigativa se genera a partir de la identificación de unos ámbitos fundamentales y que desde una concepción de integralidad pueden clasificarse en tres ámbitos: saber ser, saber conocer y saber hacer, con una interpretación holística y sistémica que los conjuga y no los admite de manera separada.

Agréguese a lo anterior que las actividades formativas se disuelven si se emprenden de manera aislada, pues el dinamismo investigativo no lo permite. En contraposición, los propósitos para la formación investigativa deben encuadrarse dentro de estrategias que consideren el currículo en su concepción compleja de procesos formativos (no como plan de estudios) y la propuesta pedagógica centrada en la alta motivación de los estudiantes.

En el caso especial de la ingeniería, un factor de especial importancia para el aprovechamiento pedagógico hacia la construcción de la competencia investigativa, es la proximidad que desde el principio tienen los estudiantes a la representación simbólica y matemática de los fenómenos y las cosas, además de las oportunidades para la experimentación que se presentan desde los primeros niveles de los planes de estudio. Además, "La concepción del ingeniero del siglo XXI representa un cambio de paradigma. El ingeniero de hoy debe ser partícipe de su propia creación; no debe buscar trabajo, sino crearlo; debe poseer una formación integral, de clase mundial, con una perspectiva y visión amplias de las realidades nacionales y mundiales; líder, de espíritu emprendedor, capaz de trabajar en equipo y sobre todo comprometido con su entorno social...." (Capote, Rizo y Bravo, 2016). Desde esta concepción de 
ingeniero, un reto para las instituciones es la creación de ambientes educativos para la formación con criterios sistémicos, donde la competencia del estudiante se vaya perfilando holísticamente desde la conjugación de experiencias investigativas y encuentros pedagógicos. Si se tiene en cuenta el anterior marco de conceptos, no hay duda que un propósito fundamental de los procesos curriculares de ingeniería, debe ser que la competencia investigativa haga parte del perfil profesional de los ingenieros, para su exhibición proactiva en sus entornos de trabajo.

Relacionado con las características del ingeniero del Siglo XXI está el propósito de formación para la innovación, cuya presencia es trascendental en las aspiraciones de competitividad y sostenibilidad empresarial. Más aun, el componente tecnológico y por eso el rol de los ingenieros, es inseparable de las iniciativas de innovación y es intrínsecamente importante por su incidencia directa en la productividad y estructura de las empresas y porque hace parte de la estrategia para lograr ventaja competitiva. Adicionalmente, la intensidad del progreso científico y tecnológico es una característica especial del desarrollo socio-económico de las naciones [3]. Recogiendo estos conceptos, es imperativo para los procesos curriculares de las facultades de ingeniería, formar profesionales que "se interesen por aprender por su propia cuenta, que adviertan la importancia de actualizarse constantemente y que sepan seleccionar e integrar el conocimiento más apropiado para el contexto en el cual desarrollan sus labores” Hernández [4].

Para comprender el concepto de competencias investigativas es menester considerar el de competencias. Los referentes al respecto son abundantes desde ángulos distintos, debido a las preocupaciones por renovaciones curriculares que atiendan realmente a los cambios sociales. En general, se han considerado dos acepciones sobre competencias, una empresarial y la otra académica. La primera enfocada hacia el tecnicismo mínimo para la producción, encaminada a definir exactamente las funciones y desempeños para el trabajo y a fijar criterios exactos para evaluar a los trabajadores. La segunda preocupada por los comportamientos que demuestran formación integral, con un abordaje más próximo a la formación integral, que lo mínimo, suficiente y necesario para el desempeño industrial. Estas competencias se han entendido de la siguiente forma:

Con un abordaje más próximo a la formación integral, que lo mínimo, suficiente y necesario para el desempeño industrial, se han entendido las competencias de la siguiente forma:

"procesos complejos que las personas ponen en acciónactuación-creación, para resolver problemas y realizar actividades (de la vida cotidiana y del contexto laboralprofesional), aportando a la construcción y transformación de la realidad, para lo cual integran el saber ser (automotivación, iniciativa y trabajo colaborativo con otros), el saber conocer (observar, explicar, comprender y analizar) y el saber hacer (desempeño basado en procedimientos y estrategias) [5]. Más concretamente, las competencias se entienden como "actuaciones integrales para identificar, interpretar, argumentar, y revolver problemas del contexto con idoneidad y ética, integrando el saber ser, el saber hacer y el saber conocer [6].

La definición de competencias así expresadas, ha dado origen a interpretaciones que ciertamente plantean la dificultad antropológica de fraccionar puntualmente en tres dimensiones los comportamientos humanos en un desempeño concreto (ser, conocer, hacer), sin embargo la postulación de tal modelo de competencias considera el criterio holístico y complejo de las actuaciones a partir de la integralidad de la persona. La división que conforma en esa triada, es solo una forma de organización de curricular.

Dentro de ese modelo tríadico (ser, hacer, conocer) puede considerarse que la competencia investigativa es un entretejido de ámbitos actitudinales, procedimentales y conceptuales. Además, se desprende también de los conceptos de Tobón y Briascos, que las competencias son manifestaciones en la acción (en el modo de interpretar las cosas y proceder en tal sentido), bajo la consideración de contextos con sus grados de imprecisión. De esta forma la competencia investigativa se forma y se manifiesta desde la actividad organizada para la indagación y el análisis de los hallazgos, donde se integran las actitudes (el espíritu investigativo), los conocimientos sobre metodología de la investigación y los procedimientos, o sea la forma como el investigador hace sus investigaciones.

Para efectos de la planeación curricular de las competencias, el modelo funcional de Tobón [5,7] incluye: descripción de la competencia, elementos de la competencia ("desempeños específicos que componen la competencia identificada”), criterios de desempeño ("son los resultados que una persona debe demostrar en situaciones reales del trabajo, del ejercicio profesional o de la vida social "), saberes esenciales ("saberes requeridos para que la persona pueda lograr los resultados.... Se clasifican en saber ser, saber conocer y saber hacer”), rango de aplicación, evidencias requeridas, problemas y casos e incertidumbres.

En el tema concreto de las competencias investigativas, estas se definen en términos de gestión del conocimiento y generación de nuevo conocimiento [8]. Se plantea que la competencia se demuestra con la utilización de las TIC Tecnologías de la Información y la Comunicación - para hacer registro de la práctica investigativa y de su contexto (capacidad para buscar, ordenar, filtrar y analizar información).

En [9] las competencias investigativas se explican de acuerdo con tres grupos: competencias del saber (cognoscitivas) como diferencias entre población y muestra, conocimientos sobre formulación de hipótesis o establecimiento de diferencias entre métodos cualitativos y cuantitativos. En un segundo grupo están las competencias del hacer como la habilidad para la delimitación del tema de investigación, la capacidad para formular acertadamente objetivos de investigación o la destreza para construir un estado del arte. En el último grupo ubica las competencias del ser, expresadas en actitudes personales como la capacidad para la crítica y la autocrítica o la habilidad para trabajar en equipos interdisciplinarios.

En [10] se hace una taxonomía similar a la anterior. Pero en el grupo de competencias del saber hacer hacen referencia a destrezas, capacidades o habilidades en tres subgrupos: capacidades cognoscitivas como el manejo crítico de la bibliografía; las destrezas motrices como la intervención con prolijidad y minuciosidad; y habilidades de comunicación como el diseño de la comunicación en ponencias o posters. 
En un estado del arte sobre formación investigativa, se concluyó que las competencias investigativas encierran un conjunto de habilidades que debe tener todo investigador tales como "el pensamiento crítico, la problematización, comunicación oral y escrita, análisis, abstracción o síntesis” [11]. Se agrega que son "competencias de alto grado de complejidad por su objeto de aprendizaje y acción”.

De otra forma, [12], define las competencias investigativas como procesos complejos que integran aprendizajes en el saber ser, saber conocer, saber hacer y saber convivir para abordar integralmente problemáticas desde lo ético y creativo, con pensamiento lógico, crítico y reflexivo frente a entorno.

Dentro del marco de los referentes anteriores, se propone en este artículo la siguiente definición de competencia investigativa: es la integración de conceptos, actitudes y procedimientos que demuestra un investigador en la planeación, desarrollo y divulgación de iniciativas para la generación y gestión de nuevo conocimiento. Esta definición que se propone considera fundamentalmente que el investigador en sus actividades demuestra conocimientos sobre metodología y sobre gestión de la información, conoce técnicas para tratamiento de datos y sabe cuál es la lógica del proceso investigativo; asimismo, demuestra predisposición, convicción, motivación y decisión personal para emprender las investigaciones. Pero no es suficiente con estos dos elementos, además organiza las secuencias acertadamente, procede con orden e iniciativa durante las etapas del proceso investigativo, sabe cómo encuadrar los informes y gestionar los recursos. Estos tres elementos los pone en acción holísticamente, como un todo, para lograr el fin de consecución de nuevo conocimiento. Dicho de otro modo, el investigador pone en escena el saber sobre el proceso investigativo sumando a su pasión por investigar y la coherencia con la que procede en sus investigaciones. Queda claro también en la definición propuesta que el ciclo de vida de las investigaciones inicia con la planeación, sigue con la administración y control de las etapas planeadas y termina con la divulgación y socialización del nuevo conocimiento. En estas tres fases entre en juego la competencia investigativa, con su carácter holístico y complejo.

La construcción de las competencias investigativas en ingeniería no es un asunto puntual que pueda considerarse independientemente. Por el contrario, su complejidad invita a que los análisis se tangan que hacer en consideración de tres direcciones: los procesos curriculares, la propuesta pedagógica y la gestión del conocimiento. Por eso es menester enfocar a continuación estos tres conceptos.

En relación con lo anterior, un primer concepto importante es el de currículo. Al respecto, en una conceptualización funcional, [13] plantea que el currículo se refiere a las siguientes preguntas ¿Para qué enseñar? (Propósitos educativos), ¿Qué enseñar? (Contenidos), ¿Cuándo enseñarlo? (Secuenciación), ¿Cómo enseñarlo? (Metodología), ¿Con qué enseñarlo? (Recursos), ¿Se cumplió o se está cumpliendo? (Evaluación). Relevancia especial se le da a la primera pregunta, al relacionarla directamente "con la finalidad, el propósito y el sentido de la educación”. Según lo anterior, es claro que el plan de estudio u organización de los contenidos es solo un componente del currículo.
Desde otro punto de vista, se dice que "el currículo asumido como plan de estudios, como conjunto de conocimientos o materias a superar por el alumno dentro de un ciclo, nivel educativo o modalidad de enseñanza, es la acepción más tradicional conocida. Además, es la más utilizada en los modelos formativos cuya esencia se basa en los procesos de transmisión, procesos en donde los limites están preestablecidos, los propósitos a lograr están predeterminados, las practicas están definidas, lo que permite dar lugar a una situación de inercia académica, que hoy es prevaleciente en muchas realidades educativas” [14]. A propósito de estas dos definiciones, vale decir que uno de los obstáculos en las reformas curriculares en las áreas de matemáticas, ciencias naturales e ingeniería y afines, es que la concepción de currículo se dirige solo a la organización del plan de contenidos (pensum), lo que lleva, por ejemplo, a que se desconozcan conexiones potenciales y reales entre practicas pedagógicas, recursos, propósitos generales y tipología de estudiantes.

En una definición más compleja, [15] considera el currículo con dos componentes: unos principios antropológicos, axiológicos, formativos, científicos, epistemológicos, metodológicos, sociológicos, psicopedagógicos, didácticos, administrativos y evaluativos y por otra parte, los medios inspirados desde estos principios para alcanzar para los procesos y propósitos de formación integral de los estudiantes y que incluyen entre otros, la organización escolar, los planes de estudio, los contenidos de la enseñanza, las estrategias didácticas y metodológicas, los espacios y tiempos para la formación multidimensional, los proyectos, los criterios e indicadores evaluativos y " los agentes educativos que intervienen, los contextos situacionales exógenos y endógenos; los recursos locativos, instrumentales, materiales y de apoyo docente”. Al segundo componente le agrega los procesos de rediseño para conseguir que con tales medios si se permitan los principios planteados. Esta definición conduce a decir que las reformas curriculares deben convocar a análisis desde la integralidad institucional desvirtuando el fraccionamiento entre el interés administrativo-financiero y el académico. En realidad, los diagnósticos y determinaciones deben considerar el currículo con visión sistémica, teniendo en cuenta que las interrelaciones de los procesos deben apuntar a los principios del proyecto educativo.

Como puede concluirse, el currículo encierra cierta totalidad educativa y esto posiblemente desprenda preocupaciones en el momento de su construcción. “¿Cómo establecer un concepto que ya casi no tiene límites en su definición? Si decimos que el campo curricular es un campo de discursos y prácticas que toman por objeto el currículo, ¿qué vamos a entender por currículo?” [14].

Otro concepto que se considera en la propuesta de competencia investigativa es el de los modelos pedagógicos, entendidos como las dinámicas que resuelven las preguntas relacionadas con el para qué, el cuándo y el con qué, en coherencia con el tipo de hombre que se quiere formar y de la concepción de sociedad que se quiera tejer [13]. Además, según [17], un modelo pedagógico es una guía para la acción educativa institucional, que centra sus intencionalidades en los intereses y necesidades de los estudiantes. De acuerdo con lo anterior, el modelo debe responder a las necesidades de 
aprendizaje con pertinencia y relevancia. Agréguese que "los modelos pedagógicos se construyen o se implementan para direccionar la educación, tener un soporte teórico les permitirá encauzar el desarrollo del proceso enseñanza -aprendizaje” [18].

La competencia investigativa encierra también la gestión del conocimiento. En relación con este concepto, para el análisis se toma como referencia el modelo KMAT de Andersen con sus funciones básicas de crear, identificar, capturar, adaptar, organizar, aplicar y compartir el conocimiento [19]. En general puede decirse que la gestión del conocimiento es el conjunto de procesos organizacionales para valorar y sistematizar la información generada por las personas. Es de aclarar que, aunque los conceptos y modelos sobre gestión del conocimiento pertenecen a ámbitos empresariales, se pueden extrapolar a la gestión de la investigación en las universidades, si se entiende esta como como una organización de proyectos, investigadores, asesores y profesores. En [20] se hace una descripción acerca de la concepción y aplicación del modelo KMAT en el caso concreto de una organización.

Para terminar los antecedentes a la propuesta, se hace una diferencia entre objeto de investigación y objeto de estudio. En [21] se plantea que un objeto de investigación es un "Constructo formal que expresa ámbitos y exigencias cognoscitivas y que media en la relación de conocimiento teorizante entre el sujeto cognoscente y el de los objetos reales” y el objeto de estudio se define allí mismo como "Cosa o fenómeno al que se enfoca el proceso de investigación, respecto del cual se formula la tesis y sobre el cual se habrán de demostrar y sostener los resultados”.

Es decir, el objeto de la investigación formaliza la relación entre el sujeto investigador y lo que se está investigando. Dicha relación expresa explícitamente los intereses del sujeto investigador para conocer ese mundo. La formalización hace referencia a la organización y planeación de los procesos de indagación y a la definición de los aspectos teóricos, contextuales y metodológicos. De acuerdo con esto, el objeto de investigación se va tejiendo con construcciones documentales incrementales como la propuesta, el anteproyecto o el informe final de resultados.

A su vez, el objeto de estudio está constituido por el foco hacia el cual se concentra la mira del investigador. Son los atributos del evento, fenómeno, cosa, que se quiere conocer. En términos más generales, es la realidad que se quiere indagar. En este sentido la delimitación de ese objeto resulta crucial para el proyecto, ya que si hay imprecisiones lo más probable es que no se tenga un buen final. En atención a esto, el equipo de investigación debe hacer el máximo esfuerzo para declarar las restricciones del objeto de estudio, acordar los aspectos que concretamente interesan, evitar vaguedades en la demarcación de la entidad que motiva la investigación. Tomando como referencia lo anterior, se concluye que la competencia investigativa involucra la integralidad de actitudes, conocimientos y procedimientos en relación con los dos constructos: el objeto de investigación y el objeto de estudio.

Como puede deducirse, la competencia investigativa involucra la integralidad de actitudes, conocimientos y procedimientos en relación con los dos constructos: el objeto de investigación y el objeto de estudio. El propósito fundamental

de los procesos curriculares de ingeniería, debe ser que la competencia investigativa haga parte del perfil profesional de los ingenieros, para su exhibición proactiva en sus entornos de trabajo.

\section{Modelo para la formación de competencia investigativa}

Teniendo en cuenta los fundamentos anteriores sobre competencias desde su enfoque holístico en la triada ser, hace y conocer; competencia investigativa, currículo en la concepción de complejidad de Iafranceso [15], pedagogía (en consideración del tipo de hombre y sociedad que se quieren formar) y gestión del conocimiento desde los fundamentos del modelo KMAT, la propuesta para la formación de investigadores en las universidades se percibe dentro de un marco complejo, en vez de un listado de propósitos y acciones puntuales. De conformidad con esto, la propuesta incluye los siguientes aspectos: la definición de la competencia investigativa y unos lineamientos relacionados con la institucionalidad curricular y la propuesta pedagógica. El modelo que se presenta en este artículo tiene una estructura así:

Tabla 1

Resumen del modelo propuesto

\section{COMPETENCIA INVESTIGATIVA}

Demostración de idoneidad en la integración de conceptos, actitudes y procedimientos para la planeación, desarrollo y divulgación de iniciativas conducentes a la generación y gestión de nuevo conocimiento en ingeniería.

\section{PROPOSITO FUNDAMENTAL}

Que la competencia investigativa haga parte del perfil profesional de los ingenieros.

ELEMENTOS DE LA COMPETENCIA INVESTIGATIVA

No 1. Actuación con ética, responsabilidad con el conocimiento, respeto y conservación de principios morales.

No 2. Conocimiento de la estructura y las interrelaciones de información de un proyecto de investigación.

No 3. Desarrollo de procesos de indagación aplicando conceptos de gestión del conocimiento.

\section{LINEAMIENTOS PARA LA FORMACIÓN DE LA} COMPETENCIA INVESTIVATIVA

No 1: Interacción de los cursos de investigación del plan de estudios de ingeniería con el contexto institucional para la investigación.

No 2: la propuesta pedagógica para la investigación formativa parte del precepto de aprender a investigar investigando.

No 3: Enfoque sistémico de los comportamientos esenciales del saber ser, el saber hacer y el saber conocer integrados en el currículo de ingeniería.

No 4: Estímulos para la investigación conectados con intereses reales de los estudiantes.

No 5: Destinación directa de recursos y definición de estrategias específicas para la formación investigativa.

No 6: Aprovechamiento de la oportunidad curricular de ingeniería para la modelación matemática y la experimentación.

No 7: Promoción de la transversalidad curricular para la formación investigativa integrando procesos en todos los niveles del plan de estudios de ingeniería.

Fuente: elaboración propia del autor 
- Una competencia investigativa desglosada en seis elementos de competencias.

- Cada elemento de competencia integrado por comportamientos esenciales: saber ser (exhibición de actitudes), saber hacer (exhibición de procedimientos) o saber conocer (exhibición de conceptos). Cada elemento considera de estos, cuales son los dominantes, no necesariamente los tres. Es decir, el elemento de competencia se enfatiza en uno o varios comportamientos esenciales.

- Un propósito fundamental

- Siete lineamientos para la construcción de la competencia investigativa en ingeniería.

Esquemáticamente el modelo se muestra en la Tabla 1.

\subsection{Competencias investigativas y sus elementos}

La competencia investigativa que demuestra el estudiante o el egresado de la facultad de ingeniería es la siguiente:

Demuestra idoneidad en la integralidad de conceptos, actitudes y procedimientos en la planeación, desarrollo y divulgación de iniciativas para la generación y gestión de nuevo conocimiento.

Elemento de competencia No 1: asume los procesos investigativos con criterios de ética, responsabilidad con el conocimiento, respeto y conservación de principios morales.

Comportamientos esperados en el ámbito esencial del saber ser:

1) Demuestra tolerancia ante las críticas y sugerencias de los demás. Acepta insuficiencia o limitaciones de sus conocimientos, razonamientos, análisis o argumentaciones y trabaja en equipos con sentido colaborativo.

2) Obtiene información y genera conocimiento con criterios de honradez y respeto por los seres vivos y los tejidos culturales, axiológicos y sociales. Asimismo, respeta la producción intelectual y científica ajena, evitando violar derechos de autoría.

3) Demuestra iniciativa propia para la indagación continua sobre problemas, soluciones, modelos o innovaciones en el campo de la ingeniería y con autonomía emprende indagaciones y explora conocimientos.

Elemento de la competencia No 2. Conoce la estructura y las interrelaciones de información de un proyecto de investigación.

Comportamientos esperados en el ámbito esencial del saber conocer:

1) Distingue técnicas, instrumentos y estrategias para obtener información, en coherencia con los objetivos e hipótesis o supuesto de proyectos de investigación.

2) Define criterios para la organización, sistematización, análisis e interpretación de la información obtenida en los procesos de indagación.

3) Comprende las concepciones de los distintos tipos de investigación y sus propósitos, para elección de las formas de acceso a la información y la organización e interpretación de los hallazgos.

Elemento de la competencia No 3. Realiza procesos de indagación de manera planeada, aplicando conceptos de gestión del conocimiento para la búsqueda, almacenamiento, transferencia, transformación, creación, organización y compartimiento de la información.

Comportamientos esperados en los ámbitos esenciales del saber hacer y el saber conocer.

1) Define con precisión el alcance del objeto de estudio del proyecto, para establecer rutas y métodos de acceso a las fuentes de información e indagación.

2) Realiza indagaciones de manera organizada e identificando los asuntos fundamentales para el logro de los objetivos del proyecto y establece criterios de búsqueda, evaluación y selección de la información y hace análisis e interpretaciones dentro de marcos de conceptualización adecuados

3) Utiliza las TIC y Crea modelos, estructuras y herramientas lógicas en procesos de búsqueda, almacenamiento, transferencia, transformación, creación, organización y compartimiento de información.

Elemento de la competencia No 4. Elabora documentos sobre planeación de proyectos de investigación e informes, formalizando la escritura de acuerdo con normas establecidas por la comunidad académica y científica de ingeniería.

Comportamientos esperados en el ámbito esencial del saber hacer:

1) Construye documentos aplicando normas de estandarización para la citación, referenciación y organización de contenidos, con criterios de orden y estética.

2) Construye documentos de acuerdo con las reglas del idioma sobre gramática, sintaxis y semántica del idioma. Con criterios de coherencia de los contenidos.

Elemento de la competencia No 5. Construye objetos de investigación considerando holísticamente situaciones problemáticas de ingeniería, fundamentos teóricos y estrategias metodológicas para la gestión del conocimiento.

Comportamientos esperados en el ámbito esencial del saber hacer.

1) Define situaciones problemáticas que conducen a la planeación y desarrollo de proyectos de investigación y relaciona la planeación de actividades con los objetivos formulados para los hallazgos y resultados del proyecto.

2) Identifica referentes teóricos y estado del arte para la fundamentación conceptual y contextual del proyecto de investigación, considerando autenticidad, validez, confiabilidad y alcance de las técnicas y las fuentes de investigación y coherencia con los objetivos del proyecto.

3) Diseña la ruta metodológica del proyecto en coherencia con los plazos, recursos, objetivos, alcances y define criterios, unidades de análisis, categorías o variables para el análisis e interpretación de la información.

Elemento de competencia No 6. Comunica los asuntos relacionados con la investigación, con criterios de claridad, organización, estética, suficiencia y procurando respeto por las personas, las comunidades y las instituciones.

Comportamientos esperados en los ámbitos esenciales del saber hacer y el saber ser.

1) Expone ideas en forma oral y de manera ordenada, con capacidad de síntesis, entonación suficiente, con argumentaciones precisas y considerando respeto por las personas, las instituciones y la sociedad. 
2) Define los criterios y canales para comunicar y transferir los resultados y hallazgos de las investigaciones y utiliza recursos de TIC para comunicación y procesamiento de información relacionada con el proceso investigativo.

3) Maneja recursos para comunicar ideas sobre el proceso investigativo utilizando lenguajes diferentes al español.

En la definición de estas competencias se ha hecho una adaptación del modelo funcional definido en $[5,12]$. Seguirlo con exactitud implicaría definir competencias, luego desglosar cada una en elementos de competencia, a continuación, asignarle a cada elemento un saber para el ser, otro para el conocer y un tercero para el hacer y además asignar criterios de desempeño. En el caso de las competencias investigativas tal segregación es inviable porque la competencia investigativa es compleja, aparece como una yuxtaposición de criterios y se demuestra con una transposición de actividades realizadas. Con esto en mente lo que se ha recogido de Tobón es definir elementos de competencia y a cada una, definirle según su naturaleza uno o varios énfasis enmarcados en los ámbitos esenciales saber ser, saber conocer o saber hacer.

\subsection{Formación de la competencia investigativa}

El desarrollo de los seis elementos de competencia es complejo debido a la multidimensionalidad de los procesos implicados y a la interactividad de los elementos que intervienen. Además, que son procesos más difusos que formalmente acotados. Con esto en mente la propuesta para su desarrollo se integra con unos lineamientos que encierran intencionalidades curriculares y pedagógicas.

1) Primer lineamiento: sobre los cursos de investigación en el plan de estudios de ingeniería.

La importancia de los cursos de asignaturas de investigación formativa (como Metodología de la Investigación) no está dentro de su ubicación en un plan de estudios de los programas de ingeniería, sino en la forma como ocurren sus interacciones con el currículo institucional, entendido este como la yuxtaposición de distintos factores institucionales y del aula, que contribuyen a la formación del estudiante. Es decir, estos cursos son aportantes en asuntos puntuales de competencias para el saber conocer, pero son insípidos si no existen articulaciones con semilleros de investigación, grupos de estudio, convocatorias de proyectos para estudiantes, eventos de divulgación o revistas para las publicaciones de los estudiantes, entro otros factores. Así que tales cursos del plan de estudios, más que enfocados en la epistemología o en la conceptualización puntual del método y la ciencia, deben es establecer vínculos vivenciales con el entorno investigativo de la institución, interno o externo. Por supuesto, tal estrategia no implica que tenga que evitarse la enseñanza de los temas concretos para las competencias del saber conocer.

2) Segundo lineamiento: sobre pedagogía para la investigación

La propuesta pedagógica para la investigación formativa parte del precepto de aprender a investigar investigando. Es decir, las competencias del ser, hacer y el conocer se forma más decididamente desde la vivencia del estudiante. En este sentido cobra vigencia la incorporación de metodologías como Aprendizaje Basado en Problemas o Aprendizaje Basado en Proyectos, que son de fácil aplicación en ingeniería Además, en los semilleros de investigación o los grupos de estudio debe partirse de fomentar el aprendizaje desde la formulación y desarrollo de proyectos. Hay que mencionar además otras estrategias como los proyectos de aula, los proyectos integradores, los proyectos de grado o los proyectos para participar en convocatorias externas o internas para estudiantes. Pero hay que llamar la atención en algo: se debe promocionar el aprendizaje y la motivación del estudiante siendo racionales con los niveles de exigencia y, sobre todo, con la pedagogía suficiente de profesores y asesores para llevar de la mano al estudiante. En síntesis, la propuesta pedagógica para la investigación formativa debe enfocarse en la alta motivación de los estudiantes y en su aprendizaje incremental desde su participación en proyectos de investigación. Tanto en la formación profesional como en los postgrados debiera de considerarse esta esencia pedagógica.

3) Tercer lineamiento: sobre la complejidad de los comportamientos esenciales

La competencia investigativa se construye desde la identificación de competencias específicas enmarcadas en comportamientos esenciales del saber ser, el saber hacer y el saber conocer. Pero, aunque en su enunciado figuran separadas, en la realidad tal segregación no es posible. Esto porque al ser la actividad investigativa una conjugación de técnicas, argumentaciones, conocimientos, normas, convivencias y comunicaciones, se concibe como una integralidad de emociones, prácticas y conocimientos. Por eso esta clasificación está pensada desde un enfoque sistémico, y desde ahí puede decirse que ninguna de los tres componentes puede formarse sin la aparición de las otras dos. Por eso los profesores y asesores tienen el compromiso de orientar a sus estudiantes en consideración de los tres, pero integradas (no importa el nivel, esto aplica tanto en la formación profesional como los postgrados). A manera de ejemplo, el tema del referenciación bibliográfica encierra asuntos de ética, respeto, conocimiento de normas técnicas y realización organizada de listas de fuentes citadas. Una oportunidad es que específicamente en ingeniería las temáticas en buena medida permiten abordajes integrados en la triada ser, conocer, hacer.

4) Cuarto lineamiento sobre los estímulos para los estudiantes

Los estímulos para los estudiantes deben evidenciarse en realidades vivas para ellos como los estipendios, becas, publicación de artículos, programación de ponencias, patrocinios para participar en redes, viajes, pasantías o premios. Es más, el estímulo debe estar conectado con los intereses reales de los estudiantes. En parte, acá entra en juego el currículo institucional, ya que desde sus procesos debe prepararse el entorno favorable por ejemplo para eventos y convocatorias de estudiantes. Además de lo anterior, los profesores investigadores son estimuladores directos o indirectos para los estudiantes. En general un currículo institucional para la investigación representada en procesos, escenarios, grupos, directrices, entre otras cosas, resulta altamente estimulante para el estudiante investigador.

5) Quinto lineamiento: sobre la asignación de recursos especiales

Tanto la investigación formativa como la formación para la investigación requieren destinación directa de recursos y definición de estrategias específicas. Por ejemplo, la asignación 
de docencia para los semilleros de investigación y grupos de interés, la programación de talleres intensivos (workshop), la obligatoriedad de incluir estudiantes en los proyectos de investigación, la destinación de espacios físicos para los estudiantes investigadores o la homologación de asignaturas del plan de estudios con actividades investigativas de los estudiantes, pueden ser opciones a considerar dentro de otras posibles.

6) Sexto lineamiento: sobre la modelación matemática y la experimentación

En el currículo de ingeniería se presenta una oportunidad relevante. Es la relacionada con la presencia de la modelación matemática y la experimentación. Estos factores pueden incidir positivamente debido al acercamiento que desde los primeros años de universidad tienen los estudiantes a la representación abstracta de los fenómenos, la utilización de simbolismos para representar la información, el rigor conceptual de las ciencias o las simulaciones en los laboratorios. Considerando lo anterior, si se establecen sinergias entre las acciones formativas de las asignaturas del plan de estudio de ingeniería con procesos y estrategias para la investigación formativa y la formación para la investigación, entonces se pueden alcanzar logros relacionados con la competencia investigativa. En este sentido, la modelación matemática es una oportunidad para que los profesores desde las clases vinculen a sus estudiantes con situaciones problemáticas que inciten en ellos preocupación por la indagación y la investigación.

7) Séptimo lineamiento: sobre la transversalidad formativa

En general, la competencia investigativa es un constructo transversal del currículo de Ingeniería. Esto significa que para apuntar hacia allá todos los procesos curriculares deben tener la preocupación por motivar la indagación en los estudiantes. En efecto, los propósitos que apuntan a estimular la imaginación de los estudiantes deben planearse tanto dentro de las asignaturas del plan de estudios así como en las actividades generales que para tal fin emprenda la institución. Además, debe comprenderse que la competencia investigativa la manifiesta el estudiante tanto en su quehacer académico como más adelante en su actividad profesional de Ingeniero. Visto de otra forma, un propósito general debe ser la planeación de la formación de la competencia investigativa como componente del perfil profesional general de los ingenieros, lo cual es posible si entran en juego las actividades pedagógicas en las clases con la naturaleza de las dinámicas y recursos institucionales

\section{Conclusiones}

Aunque es posible una disgregación de competencias específicas en clases para el saber ser, saber hacer y saber conocer, la construcción de la competencia investigativa en su globalidad implica propuestas pedagógicas que encierren la integralidad de estas desde una mirada sistémica. Es decir, pedagógicamente es inapropiado algún intento de enseñanza de un elemento de competencia en particular, si no se hacen conexiones con lo actitudinal, lo práctico y lo cognitivo. En el caso particular de la ingeniería, una oportunidad de enorme valor es los acercamientos que desde los primeros años tienen los estudiantes a la modelación de fenómenos y la representación matemática de la cotidianeidad.
Además, sin la existencia de unos procesos curriculares favorables para la investigación formativa y sin oportunidades de estímulos reales para los estudiantes, es muy difícil que cualquier intento pedagógico tenga la trascendencia esperada. Es oportuno aclarar que, desde este ángulo se concibe el currículo a partir de su complejidad institucional, que se advierte como todo aquello que incide en la formación de los estudiantes. Una mirada del currículo como simple colección de asignaturas, contenidos y objetivos de aprendizaje, resultaría insuficiente.

Finalmente, ha de apreciarse que la competencia investigativa tiene su mayor evidencia en la alta motivación del investigador. En este sentido el reto para las universidades es como incentivar a los estudiantes de formación profesional y de postgrado para que vayan más allá del cumplimiento con los proyectos de investigación por un asunto meramente coyuntural, hasta llegar a ser investigadores reconocidos por los sistemas científicos nacionales e internacionales.

\section{Referencias}

[1] Restrepo, B., Formación investigativa e investigación formativa: Acepciones y operacionalización de esta última [en línea], 2004. Disponible

http://planmaestroinv.udistrital.edu.co/documentos/PMICIUD/InvestigacionFormativa/

[2] Rodríguez, T., Rodríguez, A. y García, M., La investigación y su contribución formativa en estudiantes de las ciencias médicas, EDUMECENTRO, 8(1), pp. 43-158, 2016.

[3] Águila, A. y Tolamatl, J., Análisis de la innovación, la tecnología, los recursos humanos y la infraestructura, como impulsores de la competitividad manufacturera del sector autopartes. México: Palibrio, 2016.

[4] Hernández, G., Colmenares, J., Vargas, F., Larrahondo, J. y Guatame, P., Objetivos de los programas de formación en Ingeniería, En: Ciencia e ingeniería en la formación de ingenieros para el siglo XXI, Duque, M. Ed., Bogotá, ACOFI, pp. 59-82, 2008.

[5] Tobón, S., Formación basada en competencias. Bogotá: Ecoe Ediciones, 2005, 44 P.

[6] Briasco, I., El desafío de emprender en el Siglo XXI. Madrid: Narcea Ediciones, 2014, 19 P.

[7] Tobón, S., Formación basada en competencias. Bogotá: Ecoe Ediciones, 2013.

[8] Ministerio de Educación Nacional, Competencias TIC para el desarrollo profesional docente. Bogotá: Imprenta Nacional, 2013.

[9] Pérez, M., Fortalecimiento de las competencias investigativas en el contexto de la educación superior en Colombia, Revista de Investigaciones UNAD Bogotá, 11(1), pp. 10-34, 2012. DOI: 10.22490/25391887.770.

[10] Gayol, M., Montenegro, S., Tarrés, M. y D’Otavvio, A., Competencias investigativas. Su desarrollo en áreas de la salud, Uni-pluri/versidad, 8(2), pp. 1-8, 2008.

[11] Rojas, C. y Aguirre, S., La formación investigativa en la educación superior en América Latina y el Caribe: Una aproximación a su estado del arte. Eleuthera, 12, pp. 197-222, de 2015. DOI: 10.17151/eleu.2015.12.11.

[12] Tobón, S., La formación basada en competencias en la educación superior: El enfoque complejo. Guadalajara: Universidad Autónoma de Guadalajara, 2008.

[13] De Zubiría, J., Los modelos pedagógicos: Hacia una pedagogía dialogante. Bogotá: Cooperativa Editorial Magisterio: 2006.

[14] López, N., La de-construcción curricular. Bogotá: Cooperativa Editorial Magisterio, 2001.

[15] Iafrancesco, G., Nuevos fundamentos para la transformación curricular. Bogotá: Cooperativa Editorial del Magisterio, 2005. 
[16] Díaz, M., La evaluación curricular en el marco de la evaluación de la calidad. Contextos, 4(14), pp. 19-30, 2015.

[17] Ortiz, A., Reales, J. y Rubio, B., Ontología y epistemología de los modelos pedagógicos. Revista Educación en Ingeniería, 9(18), pp.23-34, 2014.

[18] Martínez, N., Experiencias docentes en el aula a partir del modelo pedagógico de la Universidad de La Guajira programas de extensión Villanueva. Escenarios, 12(2), pp. 86-95, 2014. DOI: 10.15665/esc.v12i2.317

[19] Freitas, V., Modelo holístico de sistema de gestión del conocimiento para las instituciones de educación superior. Enl@ce: Revista Venezolana de Información, Tecnología y Conocimiento, 11(3), pp. 123-154, 2014.

[20] Saryazdi, M. and Owlia, M., Analysis of knowledge management effectiveness on business excellence using system dynamica. Production and Operations Management, 5(1), pp. 41-55, 2014.

[21] Ortiz, F., Diccionario de metodología de la investigación científica. México: Limusa, 2013.
J.E. Parra-Castrillón, es Ing. de Sistemas de la Universidad de Antioquia y Lic. en Matemáticas. MSc. en Software Libre, MSc. en Educación, Esp. en Docencia, Esp. en Pedagogía de la Virtualidad. Catedrático de la Universidad de Antioquia y del Politécnico Jaime Isaza Cadavid. Docente de tiempo completo de UNISABANETA. Desde 1993 ha desempeñado funciones de gestión académica, investigación y docencia en Instituciones de Educación Superior del Departamento de Antioquia.

ORCID: 0000-0002-5623-7440 